\title{
Chemical Reactivity
}

\section{FOREWORD}

Considering the importance of chemical reactivity and its strong foundations within a conceptual density functional theory (DFT) framework, the Indian Academy of Sciences, Bangalore decided to bring out a Special Issue of the Journal of Chemical Sciences on "Chemical Reactivity". Leading experts in this field from across the globe have contributed papers on diverse aspects of reactivity theory.

These papers cover the whole gamut of topics within a DFT parlance encompassing different systems such as clay-type inorganic materials, boron porphyrin complexes, zeolites and hypervalent silicon compounds, as well as various concepts such as electron localization function, molecular quantum similarity, condensed atomic indices, Coulomb holes, reactant resolution, higher order energy derivatives, excitation energy, charge sensitivity, reaction force, electron propagators, separability and $N$-representability. There are also contributions which deal with intramolecular hydrogen shifts, cycloaddition reactions, interactions among toxins and biosystems, and electronegativity equalization.

The special issue is adorned with two papers (one in the beginning and the other at the end) contributed by Ralph G Pearson and Robert G Parr. Pearson's article gives a personal account of the evolution of the hardness concept; Parr in his article has set out fourteen problems in DFT.

I thank the Indian Academy of Sciences, and in particular, the Editorial Board of the Journal of Chemical Sciences for inviting me to be the Guest Editor of this special issue. I am also grateful to the authors and the reviewers for their active support and cooperation. 